\title{
Fully differential cross sections for electron-impact excitation-ionization of aligned $D_{2}$
}

\author{
E. Ali, ${ }^{1}$ A. L. Harris,${ }^{2}$ J. Lower ${ }^{3}$ E. Weigold ${ }^{3}$ C. G. Ning, ${ }^{4}$ and D. H. Madison ${ }^{1}$ \\ ${ }^{1}$ Missouri University of Science and Technology, Rolla, Missouri 65409, USA \\ ${ }^{2}$ Illinois State University, Normal, Illinois 61761, USA \\ ${ }^{3}$ The Australian National University, Canberra ACT 0200, Australia \\ ${ }^{4}$ Department of Physics and State Key Laboratory of Low-Dimensional Quantum Physics, Tsinghua University, Beijing 100084, China
}

(Received 7 April 2014; published 23 June 2014)

\begin{abstract}
We examine fully differential cross sections for $176 \mathrm{eV}$ electron-impact dissociative excitation-ionization of orientated $\mathrm{D}_{2}$ for transitions to final ion states $2 s \sigma_{g}, 2 p \sigma_{u}$, and $2 p \pi_{u}$. In previous work [Phys. Rev. A 88, 062705 (2013)], we calculated these cross sections using the molecular four-body distorted wave (M4DW) method with the ground-state $\mathrm{D}_{2}$ wave function being approximated by a product of two Dyson $1 s$-type orbitals. The theoretical results were compared with experimental measurements for five different orientations of the target molecule (four in the scattering plane and one perpendicular to the scattering plane). For the unresolved $2 s \sigma_{g}+2 p \pi_{u}$ final states, good agreement with experiment was found for two of the five measured orientations, and for the $2 p \sigma_{u}$ final state, good agreement was found for three of the five orientations. However, theory was a factor of 200 smaller than experiment for the $2 p \sigma_{u}$ state. In this paper, we investigate the importance of the approximation for the molecular ground-state wave function by repeating the M4DW calculation using a better variational wave function for the ground state.
\end{abstract}

DOI: 10.1103/PhysRevA.89.062713

PACS number(s): $34.80 . \mathrm{Dp}$

\section{INTRODUCTION}

One of the fundamental unsolved problems in physics is the few-body problem which arises from the fact that the Schrödinger equation can only be solved analytically for two interacting particles. Consequently, for three or more particles, theoretical approximations must be made and the validity of these approximations can only be checked by comparing with experiment. In the past couple of decades, there have been numerous studies of the effective three-body problem and significant progress has been made in terms of agreement between experiment and theory, especially for ionization of the smaller atoms [see, for example, the convergent close-coupling (CCC) method by Bray and Stelbovics [1], the complex exterior scaling (ECS) technique by Rescigno et al. [2], or the time-dependent close-coupling method by Colgan et al. [3]].

For high-energy incident electrons, the collision takes place so fast that the interactions between the free particles and the target are not important and plane waves can be used to represent the free particles. In this case, the initial bound-state wave function of the target determines the outcome of the collision and the $T$ matrix becomes the Fourier transform of the target coordinate space wave function which is the momentum space wave function. Consequently, measuring the cross section becomes equivalent to measuring the momentum space wave function [4]. For lower incident-electron energies, one cannot ignore the interactions between the free electrons and the target or the final-state interactions between the projectile and ejected electron. In this case the dynamics becomes important and measuring these cross sections represents a more sensitive test of the theoretical models. For collisions with molecules, most of the measurements do not determine the orientation of the molecule so theories have to average over all orientations, and any averaging procedure can potentially mask important physics, so the most sensitive test of theory would be measuring cross sections which determine the orientation of the molecule. The first measurement of this type was performed by Takahashi et al. [5] but the statistics were not very good.

One way to determine the alignment of the molecule is to measure one of the fragments of dissociation since the fragments leave the molecule in a straight line along the direction of alignment. The excited states of $\mathrm{H}_{2}$ will immediately disassociate and the ground state can disassociate. The first experiment with better statistics was measured by Dorn's group $[6,7]$ and they looked at ground-state dissociation. We have recently reported an experiment which determined the alignment by looking at dissociation of the excited state [8,9]. This measurement represents a stringent test of theory since there are two active electrons (four-body problem). To date, there have been a limited number of studies reported of the four-body problem for electron-impact excitation-ionization of atoms [10-14] and molecules [4,5,8,9]. For excitationionization of helium, although there was some qualitative shape agreement between experiment and theory, overall the agreement was not very good [5].

In the past few years, there have been several papers comparing experiment and the molecular three-body distorted wave (M3DW) for electron-impact ionization molecules for cases where the target orientation is not determined in the experiment [15-18]. In the early work, an approximation called the orientation-averaged molecular orbital (OAMO) was made [19] which greatly reduced the computer demands, and this approximation worked well for ionization of $\mathrm{H}_{2}$ [20-23] but not so well for the larger molecules [6,7,21-27]. Very recently, the computer codes were parallelized such that proper averages over orientations can be performed and the agreement between experiment and theory was greatly improved for the larger molecules [28].

As mentioned above, the orientation of the molecule can be determined by either looking at the dissociation of the ground state or the excited state. For ground-state dissociation, there is only one active target electron and the problem can be treated as a three-body problem. For this case, good 
agreement between experiment and theory is found for both the M3DW and the time-dependent close-coupling (TDCC) approximations [6,7,21-27]. In the second type of experiment, the residual target electron is excited and the excited-state ion will disassociate. This type of experiment requires a four-body theoretical approach, and very recently we compared the results of the molecular four-body distorted wave (M4DW) with the Canberra measurements [9] for excitation-ionization of $\mathrm{D}_{2}$. In this measurement, the $2 p \sigma_{u}$ excited state was energetically resolved while the $2 s \sigma_{g}$ and $2 p \pi_{u}$ states could not be energetically resolved from one another due to their common dissociation limit, which meant that we needed to calculate cross sections for $2 s \sigma_{g}+2 p \pi_{u}$ to compare with experiment.

The theoretical results were compared with experimental measurements for five different orientations of the target molecule (four in the scattering plane and one perpendicular to the scattering plane). For the unresolved $2 s \sigma_{g}+2 p \pi_{u}$ final states, good agreement with experiment was found for two of the five measured orientations, and for the $2 p \sigma_{u}$ final state the magnitude of the theory was much smaller than experiment. However, excellent shape agreement was found for three of the five orientations. In the theoretical calculation, the ground-state wave function for $\mathrm{D}_{2}$ was approximated as a product of two $1 s$-type Dyson orbitals. In this paper, we investigate the importance of the approximation for the molecular ground-state wave function by repeating the M4DW calculation using a better variational wave function for the ground state.

\section{METHOD OF CALCULATION}

In this paper, we have used the molecular four-body distorted wave (M4DW) approach, which is described more fully in Ref. [29]. Since the collision time is much shorter than the vibrational or rotational times, we make the usual assumption of stationary nuclei. For the four-body problem, the $T$ matrix is a nine-dimensional (9D) integral which we evaluate numerically,

$$
T_{f i}=\left\langle\psi_{f}\left|V_{i}-U_{i}\right| \phi_{i}\right\rangle .
$$

Here $\phi_{i}$ is the initial-state wave function which we express as

$$
\left|\phi_{i}\right\rangle=\left|\psi_{\text {target }}\left(\mathbf{r}_{1}, \mathbf{r}_{2}\right) \chi_{i}^{+}\left(\mathbf{k}_{i}, \mathbf{r}_{0}\right)\right\rangle .
$$

Here $\chi_{i}^{+}\left(\mathbf{k}_{i}, \mathbf{r}_{0}\right)$ is a continuum-state distorted wave for wave number $\mathbf{k}_{\mathbf{i}}$ and the + indicates outgoing wave boundary conditions. In our previous work, we approximated the ground-

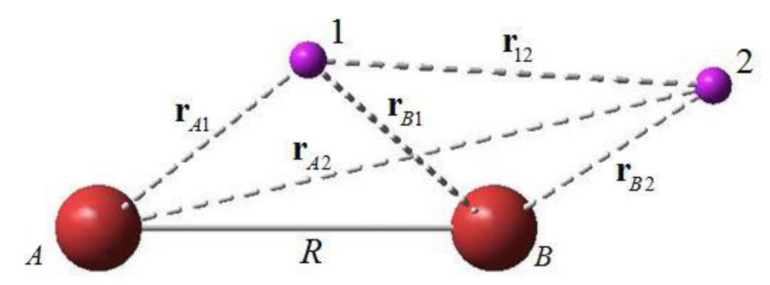

FIG. 1. (Color online) Coordinates used in the Rosen wave function [30].

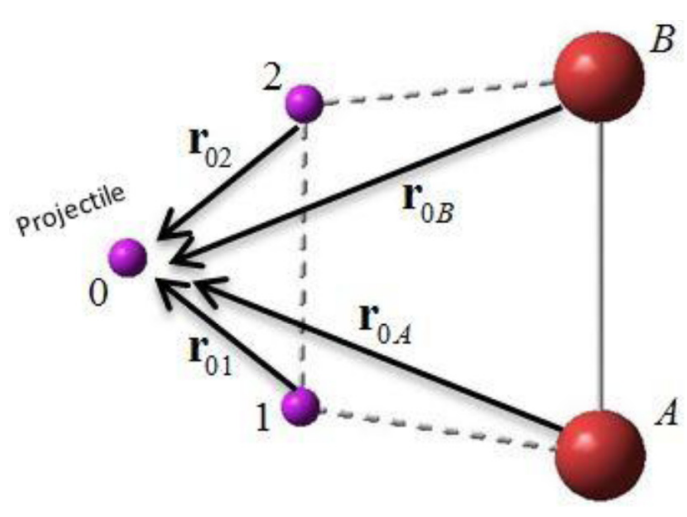

FIG. 2. (Color online) Coordinates for the initial-state interaction potential.

state wave function for the target $\psi_{\text {target }}\left(\mathbf{r}_{1}, \mathbf{r}_{2}\right)$ as a product of two Dyson $1 s$-type orbitals. In this work, we use the variational wave function of Rosen [30] which contains both $s$ - and $p$-state contributions. For this wave function, the dissociation energy was within $10 \%$ of the experimental value, which represents a significant improvement over the product of Dyson orbitals. There are more complicated wave functions for $\mathrm{H}_{2}$ which give even better energies but we found that, in the evaluation of a
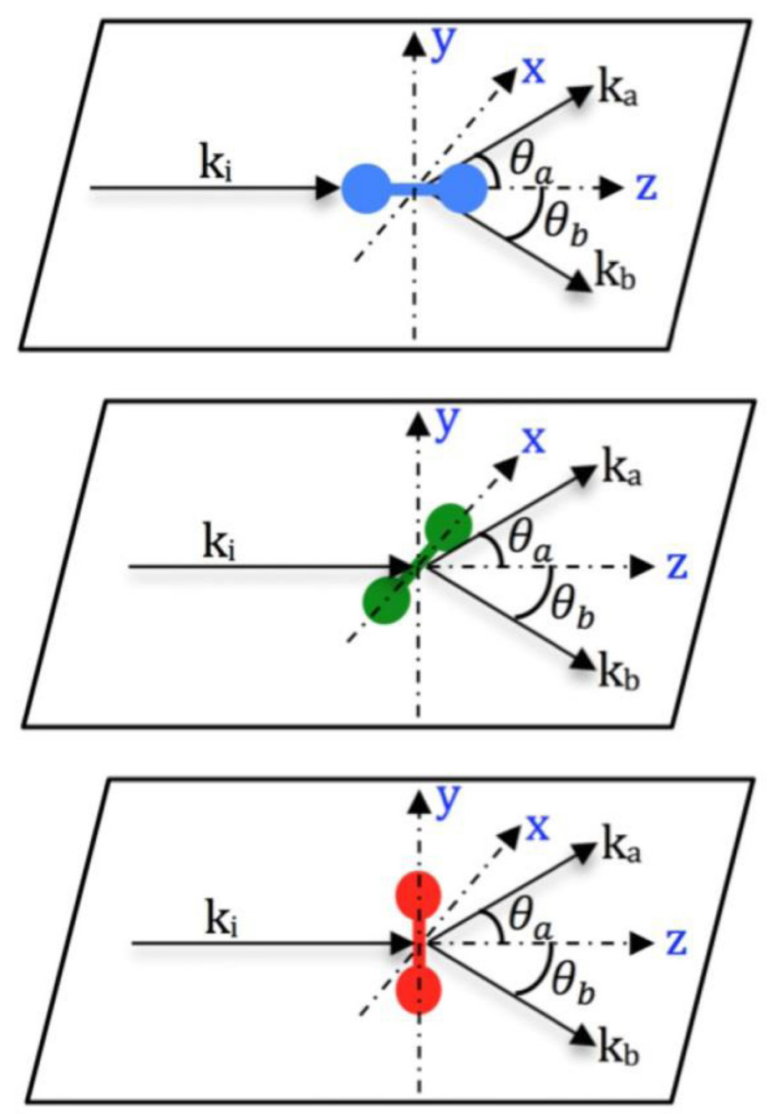

FIG. 3. (Color online) Three of the measured orientations of the deuterium molecule. The wave number of the incident electron is $\mathbf{k}_{i},\left(\mathbf{k}_{a}, \theta_{a}\right)$ are the wave number and scattering angle for the faster final-state electron, and $\left(\mathbf{k}_{b}, \theta_{b}\right)$ are the wave number and scattering angle for the slower final-state electron. 
9D integral, the time required to evaluate the ground-state wave function was crucial to the feasibility of evaluating the integral. For example, we tried a 50-term and a 30-term Hartree-Fock (HF) ground-state wave function and quickly learned that it was not feasible to use these wave functions. The calculations presented here using the Rosen wave function required $2000000 \mathrm{SU}$ (Scalable Units) on the Extreme Science and Engineering Discovery Environment (XSEDE) cluster (Kraken) while we estimated that the HF wave functions would require several hundred million SU on the same cluster, which is obviously not feasible. The Rosen wave function can be expressed as

$$
\psi_{\mathrm{target}}=N\left[\phi_{A 1}\left(\mathbf{r}_{A 1}\right) \phi_{B 2}\left(\mathbf{r}_{B 2}\right)+\phi_{A 2}\left(\mathbf{r}_{A 2}\right) \phi_{B 1}\left(\mathbf{r}_{B 1}\right)\right],
$$

where $N$ is the normalization factor, $A$ and $B$ denote the two nuclei for the $\mathrm{D}_{2}$ molecule, and $\left(r_{A 1}, r_{B 2}\right)$ are the distance of electrons 1 and 2 from the nuclei as shown in Fig. 1.

The trial wave function $\phi_{A 1}$ is expressed as a linear combination of a $1 s$ and $2 p_{z}$ wave function,

$$
\phi_{A 1}\left(\mathbf{r}_{A 1}\right)=N_{0}\left\{\phi_{1 s}\left(\mathbf{r}_{A 1}\right)+\sigma \phi_{2 p z}\left(\mathbf{r}_{A 1}\right)\right\} .
$$
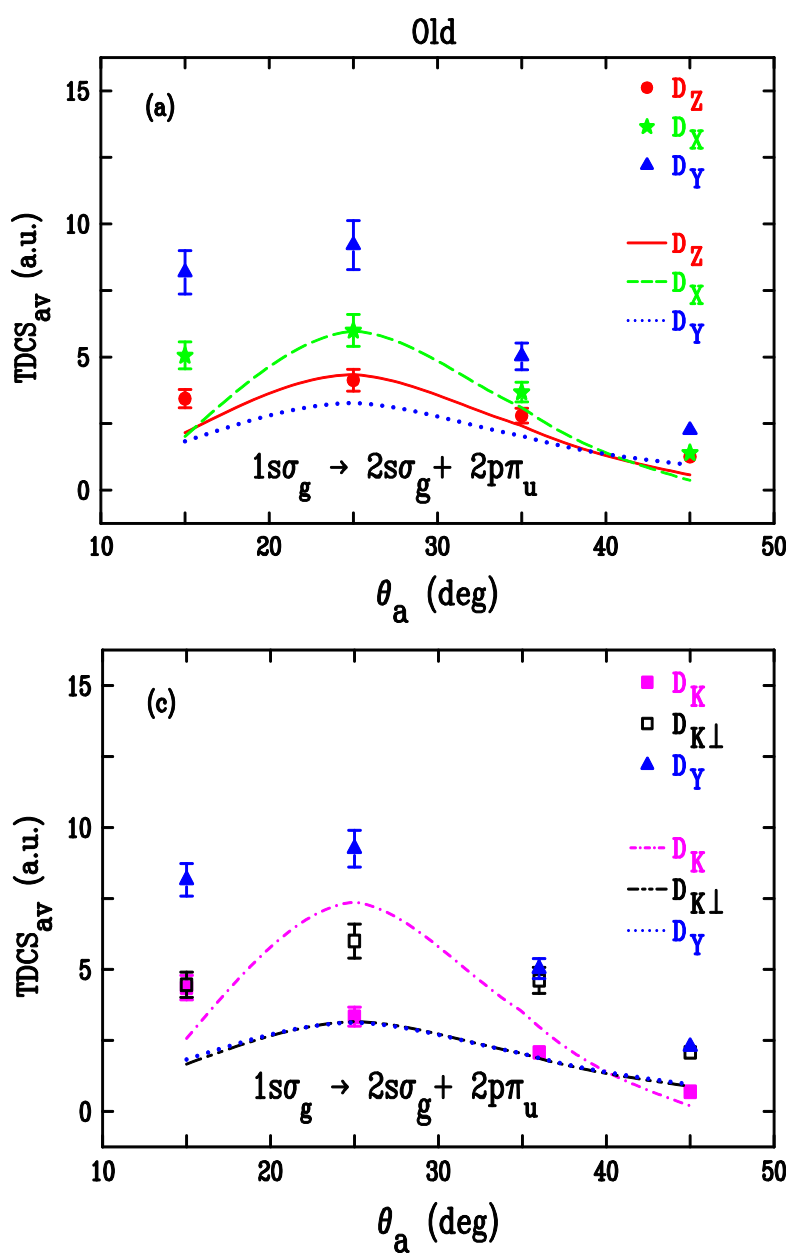

Here $\sigma$ is a parameter to minimize the energy, $N_{0}$ is the normalization factor, and we use the values obtained by Rosen [30].

The final-state wave function $\psi_{f}$ in the $T$ matrix of Eq. (1) is approximated as follows:

$$
\left|\psi_{f}\right\rangle=\left|\chi_{a}^{-}\left(\mathbf{k}_{a}, \mathbf{r}_{0}\right) \chi_{b}^{-}\left(\mathbf{k}_{b}, \mathbf{r}_{1}\right) \phi_{\text {ion }}\left(\mathbf{r}_{2}\right) C_{a-b}\left(\mathbf{r}_{01}\right)\right\rangle .
$$

Here $\chi_{a}^{-}\left(\mathbf{k}_{a}, \mathbf{r}_{0}\right)$ is the final-state distorted wave function for a scattered projectile with wave number $\mathbf{k}_{a}, \chi_{b}^{-}\left(\mathbf{k}_{b}, \mathbf{r}_{1}\right)$ is the distorted wave function for the ejected electron, the $(-)$ indicates incoming wave boundary conditions, $\phi_{\text {ion }}\left(\mathbf{r}_{2}\right)$ is the excited-state wave function for the final-state ion which is a Dyson wave function, and $C_{a-b}\left(\mathbf{r}_{01}\right)$ is the Coulomb interaction between the two outgoing electrons.

The perturbation in Eq. (1) contains the initial-state interaction potential $V_{i}$ between the projectile electron and target is given by

$$
V_{i}=-\frac{1}{r_{0 A}}-\frac{1}{r_{0 B}}+\frac{1}{r_{01}}+\frac{1}{r_{02}} .
$$

Here $r_{01}, r_{02}$ are the distances between the projectile electron and the two bound electrons of the $\mathrm{D}_{2}$ molecule, and $r_{0 A}$ and $r_{0 B}$ are the distances between the projectile electron and the
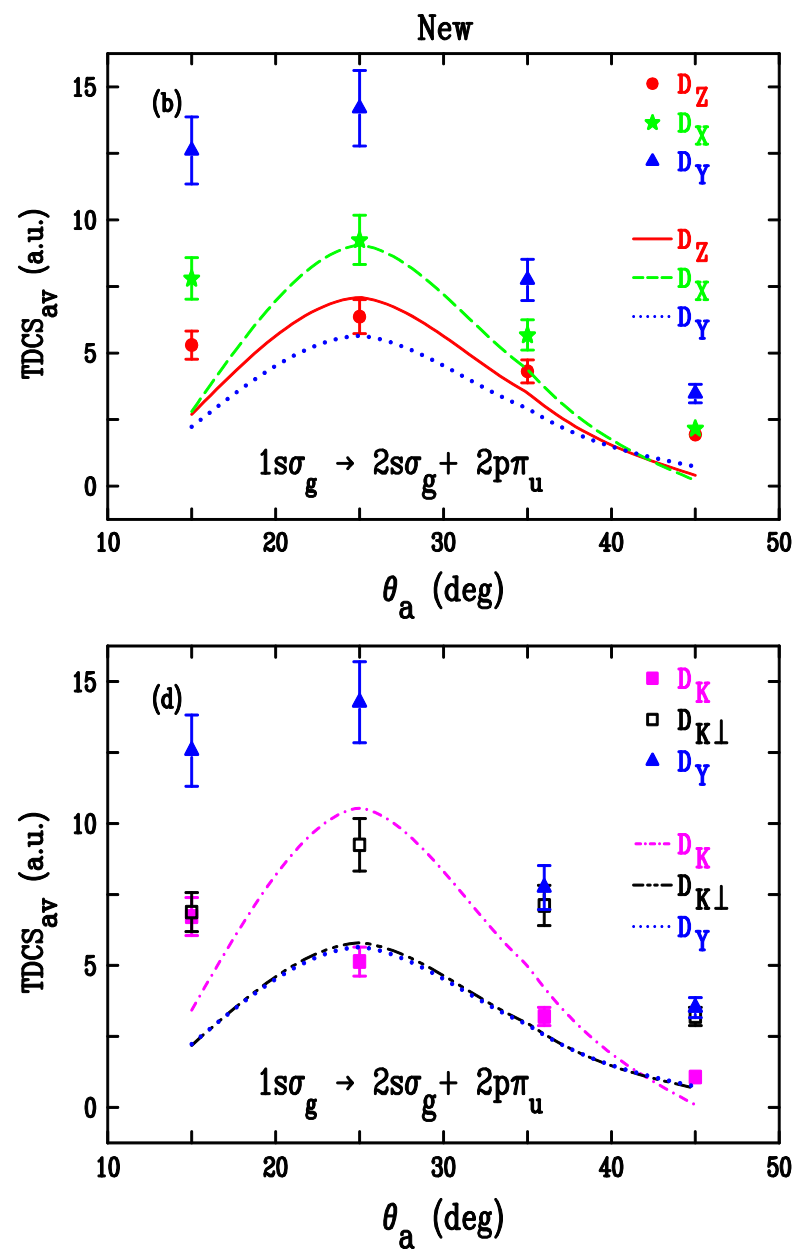

FIG. 4. (Color online) Triple differential cross sections (TDCSs) for electron-impact ionization of aligned molecular $\mathrm{D}_{2}$. Experimental results are from Ref. [9]. The figure contains a comparison of theory and experiment for the old theoretical results from Ref. [9] obtained using a product of Dyson wave functions for the ground state of $\mathrm{D}_{2}[(\mathrm{a})$ and (c)] and the new results obtained using the Rosen wave function [(b) and (d)]. The different molecular orientations are described in the text. The scattering angle for the faster final-state electron is $\theta_{a}$ and the ejection angle for the slower final-state electron is averaged over an angular range of $40^{\circ}-80^{\circ}$. 
two nuclei, as shown in Fig. 2. The final term in the perturbation of Eq. (1) is $U_{i}$, which is an initial-state spherically symmetric approximation for $V_{i}$.

Combining all our approximations, the M4DW $T$ matrix [29] can be written as

$$
\begin{aligned}
T_{f i}= & \left\langle\chi_{a}^{-}\left(\mathbf{k}_{a}, \mathbf{r}_{0}\right) \chi_{b}^{-}\left(\mathbf{k}_{b}, \mathbf{r}_{1}\right) \phi_{\text {ion }}\left(\mathbf{r}_{2}\right) C_{a-b}\left(\mathbf{r}_{01}\right)\right. \\
& \left.\times\left|V_{i}-U_{i}\right| \psi_{\text {target }}\left(\mathbf{r}_{1}, \mathbf{r}_{2}\right) \chi_{i}^{+}\left(\mathbf{k}_{i}, \mathbf{r}_{0}\right)\right\rangle
\end{aligned}
$$

In terms of computer time, the calculation of the wave functions and Coulomb interactions takes very little time and can basically be ignored compared to the time required for the 9D integral, so this is the part of the code we parallelized. The 9D integral is 9-nested DO loops and the number of available processors determines which loop we use for parallelization.

\section{RESULTS}

Simultaneous measurements were performed under identical experimental conditions for three orthogonal molecular orientations described in two different Cartesian coordinate systems (see Ref. [9] for details). Figure 3 shows the three different measured orientations for the $\mathrm{D}_{2}$ molecule in one of the systems: (a) parallel to the incident beam ( $z$ axis); (b) perpendicular to the incident beam and in the scattering plane ( $x$ axis); and (c) perpendicular to the incident beam and perpendicular to the scattering plane ( $y$ axis). Both final-state electrons were detected in the scattering plane ( $x z$ plane), with $\mathbf{k}_{a}\left(\theta_{a}\right)$ being the wave number and scattering angle of the faster final-state electron and $\mathbf{k}_{b}\left(\theta_{b}\right)$ being the wave number and scattering angle of the slower final-state electron

Figure 4 compares the old and new results for excitation of the $2 s \sigma_{g}$ state. The top half of the figure contains theory and experiment for three different measured orientations for the $\mathrm{D}_{2}$ molecule: (1) parallel to the incident beam ( $z$ axis labeled $\left.D_{Z}\right)$; (2) perpendicular to the incident beam and in the scattering plane ( $x$ axis labeled $D_{X}$ ); and (c) perpendicular to the incident beam and perpendicular to the scattering plane ( $y$ axis labeled $D_{Y}$ ). The bottom half of Fig. 4 contains theory and experiment for a different set of three mutually perpendicular orientations for the $\mathrm{D}_{2}$ molecule: (1) parallel to the momentum transfer direction (labeled $D_{K}$ ); (2) perpendicular to the momentum
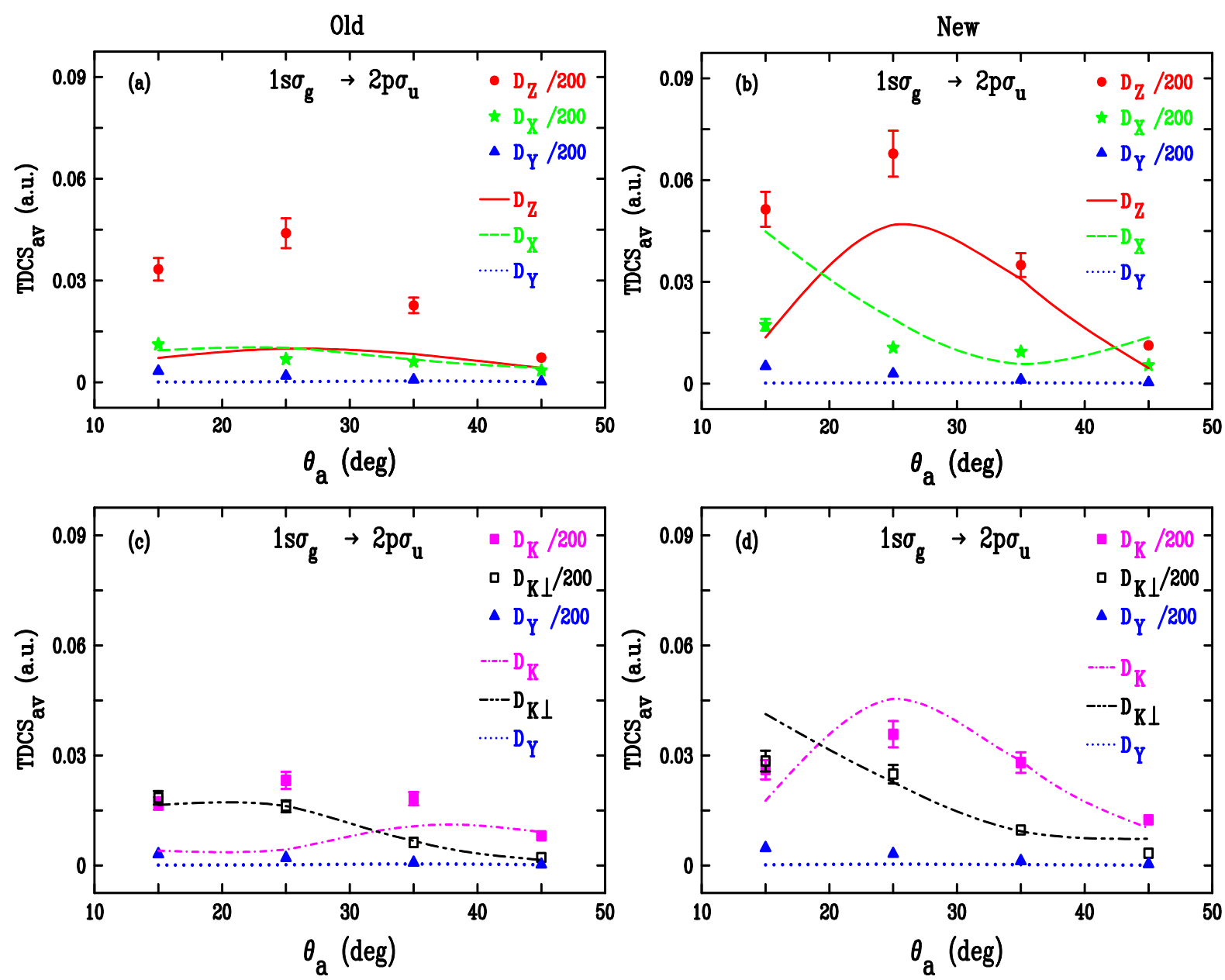

FIG. 5. (Color online) Triple differential cross sections (TDCSs) for electron-impact ionization of aligned molecular $\mathrm{D}_{2}$. Experimental results are from Ref. [9]. The figure contains a comparison of theory and experiment for the old results obtained using a product of Dyson wave functions for the ground state of $\mathrm{D}_{2}$ and the new results obtained using the Rosen wave function. The different molecular orientations are described in the text. The scattering angle for the faster final-state electron is $\theta_{a}$ and the ejection angle for the slower final-state electron is averaged over an angular range of $40^{\circ}-80^{\circ}$. 
transfer direction and in the scattering plane (labeled $D_{K \perp}$ ); and (c) perpendicular to the scattering plane ( $y$ axis labeled $\left.D_{Y}\right)$. Both final-state electrons were detected in the scattering plane ( $x z$ plane) with $\mathbf{k}_{a}\left(\theta_{a}\right)$ being the wave number and scattering angle of the faster final-state electron and the ejection angle for the slower final-state electron is averaged over an angular range of $40^{\circ}-80^{\circ}$.

The experiments were performed for exciting the unresolved $\left(2 s \sigma_{g}+2 p \pi_{u}\right)$ states. However, in Ref. [9], we found that the $2 p \pi_{u}$ state made a negligible contribution and could be ignored. Consequently, since these calculations are very computationally expensive, we only calculated results for the $2 s \sigma_{g}$ state using the new Rosen wave function. The results are for an incident-electron energy of $178 \mathrm{eV}$, fast and slow electron energies of 101.5 and $37.4 \mathrm{eV}$, respectively, and for varying $\theta_{a}$ from $15^{\circ}$ to $45^{\circ}$. The direction of molecular orientation was determined by assuming that the molecular ion fragments leave the collision in the same direction as the molecular orientation [9]. The experimental measurements were performed simultaneously under identical experimental conditions, which means that a single normalization will place all the measured data on an absolute scale and we have normalized the experiment to theory for excitation of the $2 s \sigma_{g}$ state, $\theta_{a}=25^{\circ}$, and $D_{X}$ orientation. The absolute values of the old and new cross sections are different at this point since the new wave function gave a somewhat larger cross section for this point. As can be seen from Fig. 4, there is very little difference between the old and new results in terms of agreement between experiment and theory for excitation of the $2 s \sigma_{g}$ state. We attribute the fact that there is little difference between the results of two different ground-state wave functions to the nearly symmetrical symmetry of the excited state.

It is interesting to note that there is very good agreement between experiment and theory (both shape and relative magnitude) for $D_{X}$ and $D_{Z}$ which are both in the scattering plane while the agreement is not good for the other two in-scattering-plane measurements $D_{K}$ and $D_{K \perp}$. In fact, experiment and theory do not even agree on which cross section is largest for $D_{K}$ and $D_{K \perp}$. This is quite different from ionization of the ground state of $\mathrm{H}_{2}$, where both experiment and theory found the largest cross sections for the $D_{K}$ orientation [6] while here theory still finds the largest cross section for $D_{K}$ while experiment finds the smallest cross sections for $D_{K}$. It is also interesting to note that experiment finds the largest cross sections for the $D_{Y}$ orientation while theory finds this the smallest cross section (even zero for the $2 p \sigma_{u}$ state see below).

Figure 5 compares experiment with old and new theoretical fully differential cross sections (FDCSs) for electron-impact dissociative excitation-ionization of the $2 p \sigma_{u}$ state for the same orientations shown in Fig. 5. The experimental data have been normalized for the $2 p \sigma_{u}$ state the same as described above. With this normalization, both the old and new theoretical calculations are a factor of 200 smaller than the experiment (obviously we could have normalized the experiment to the theoretical $2 p \sigma_{u}$ state which would have made theory 200 times larger than experiment for the $2 s \sigma_{g}$ state). As can be seen from the figure, the shape agreement between experiment and theory is significantly better for the Rosen ground-state wave function. Except for the smallest $\theta_{a}$, the relative magnitudes and shapes of the various theoretical orientations are in good agreement with experiment. For this state, the cross section for the $D_{Y}$ orientation is zero for both ground states due to the symmetry of the $2 p \sigma_{u}$ state. It seems a bit odd that theory is in much better agreement with experiment for the small $2 p \sigma_{u}$ cross sections than for the dominant $2 s \sigma_{g}$ cross sections.

\section{CONCLUSIONS}

In conclusion, we have previously reported a M4DW calculation for electron-impact excitation-ionization of molecular $\mathrm{D}_{2}$ using an elementary product of two Dyson orbitals to approximate the ground-state wave function. In comparison with experiment, we found good agreement for approximately $2 / 3$ of the measured cases and poor to bad agreement for the rest. In this paper, we examined the importance of the quality of the ground-state wave function by repeating the calculation with a variational wave function containing both $s$ - and $p$-state components.

Interestingly, for excitation of the dominant $2 s \sigma_{g}$ state, we found that the results were almost the same using the better wave function. However, for exciting the weaker $2 p \sigma_{u}$ final state, our M4DW results were in good agreement with all the measured data points (shape and relative magnitude) except for a projectile scattering angle of $15^{\circ}$ (the smallest angle measured). In spite of the improvement brought about by implementation of a superior ground-state wave function, the large disparity (around a factor of 200) between the predictions of theory and experiment for the strength of the transition to the $2 p \sigma_{u}$ state relative to that for the combined $2 s \sigma_{g} / 2 p \pi_{u}$ states remains.

\section{ACKNOWLEDGMENTS}

E.A. and D.M. acknowledge support of the U.S. National Science Foundation under Grant No. PHY-1068237 and XSEDE resources provided [31] by the Texas Advanced Computing Center (Grant No. TG-MCA07S029).
[1] I. Bray and A. T. Stelbovics, Phys. Rev. A 46, 6995 (1992).

[2] T. N. Rescigno, M. Baertschy, W. A. Isaacs, and C. W. McCurdy, Science 286, 2474 (1999).

[3] J. Colgan, M. S. Pindzola, and F. Robicheaux, J. Phys. B: At. Mol. Opt. Phys. 37, L377 (2004).

[4] E. Weigold and I. E. McCarthy, Electron Momentum Spectroscopy (Kluwer Academic/Plenum, New York, 1999).
[5] M. Takahashi, N. Watanabe, Y. Khajuria, Y. Udagawa, and J. H. D. Eland, Phys. Rev. Lett. 94, 213202 (2005).

[6] A. Senftleben, O. Al-Hagan, T. Pflüger, X. Ren, D. Madison, A. Dorn, and J. Ullrich, J. Chem. Phys. 133, 044302 (2010).

[7] X. Ren, T. Pflüger, S. Xu, A. Senftleben, J. Colgan, M. S. Pindzola, A. Dorn, and J. Ullrich, J. Phys.: Conf. Ser. 388, 052037 (2012). 
[8] S. Bellm, J. Lower, E. Weigold, and D. W. Mueller, Phys. Rev. Lett. 104, 023202 (2010).

[9] J. C. A. Lower, E. Ali, S. Bellm, E. Weigold, A. Harris, C. G. Ning, and D. Madison, Phys. Rev. A 88, 062705 (2013).

[10] G. Sakhelashvili, A. Dorn, C. Höhr, J. Ullrich, A. S. Kheifets, J. Lower, and K. Bartschat, Phys. Rev. Lett. 95, 033201 (2005).

[11] J. Lower, R. Panajotović, S. Bellm, and E. Weigold, Rev. Sci. Instrum. 78, 111301 (2007).

[12] S. Bellm, J. Lower, K. Bartschat, X. Guan, D. Weflen, M. Foster, A. L. Harris, and D. H. Madison, Phys. Rev. A 75, 042704 (2007).

[13] S. Bellm, J. Lower, E. Weigold, I. Bray, D. V. Fursa, K. Bartschat, A. L. Harris, and D. H. Madison, Phys. Rev. A 78, 032710 (2008).

[14] A. L. Harris, B. Milum, and D. H. Madison, Phys. Rev. A 84, 052718 (2011).

[15] O. Al-Hagan, D. H. Madison, and C. Ning, J. Phys. B: At., Mol. Opt. Phys. 43, 035201 (2010).

[16] D. H. Madison and O. Al-Hagan, J. At., Mol., Opt. Phys. 2010, 367180

[17] D. B. Jones, J. D. Builth-Williams, S. M. Bellm, L. Chiari, H. Chaluvadi, D. H. Madison, C. G. Ning, B. Lohmann, O. Ingólfsson, and M. J. Brunger, Chem. Phys. Lett. 572, 32 (2013).

[18] J. D. Builth-Williams, S. M. Bellm, L. Chiari, P. A. Thorn, D. B. Jones, H. Chaluvadi, D. H. Madison, C. G. Ning, B. Lohmann, G. da Silva, and M. J. Brunger, J. Chem. Phys. 139, 034306 (2013).
[19] J. L. Peacher and D. H. Madison, J. Chem. Phys. 123, 204302 (2005).

[20] O. Al-Hagan, C. Kaiser, D. Madison, and A. J. Murray, Nat. Phys. 5, 59 (2009).

[21] J. Colgan, O. Al-Hagan, D. H. Madison, C. Kaiser, A. J. Murray, and M. S. Pindzola, Phys. Rev. A 79, 052704 (2009).

[22] O. Al-Hagan, A. J. Murray, C. Kaiser, J. Colgan, and D. H. Madison, Phys. Rev. A 81, 030701 (2010).

[23] J. Colgan, M. S. Pindzola, F. Robicheaux, C. Kaiser, A. J. Murray, and D. H. Madison, Phys. Rev. Lett. 101, 233201 (2008).

[24] A. Senftleben, T. Pflüger, X. Ren, O. Al-Hagan, B. Najjari, D. Madison, A. Dorn, and J. Ullrich, J. Phys. B: At., Mol. Opt. Phys. 43, 081002 (2010).

[25] K. Nixon, A. Murray, H. Chaluvadi, S. Amami, D. H. Madison, and C. Ning, J. Chem. Phys. 136, 094302 (2012).

[26] X. Ren, T. Pflüger, A. Senftleben, C. G. Ning, S. Yan, P. Zhang, J. Yang, X. Ma, J. Ullrich, D. H. Madison, and A. Dorn, J. Chem. Phys. 137, 024301 (2012).

[27] X. Ren, T. Pflüger, S. Xu, J. Colgan, M. S. Pindzola, A. Senftleben, J. Ullrich, and A. Dorn, Phys. Rev. Lett. 109, 123202 (2012).

[28] H. Chaluvadi, C. G. Ning, and D. Madison, Phys. Rev. A 89, 062712 (2014).

[29] A. L. Harris, M. Foster, J. L. Peacher, and D. H. Madison, J. Phys. B 41, 135203 (2008).

[30] N. Rosen, Phys. Rev. 38, 2099 (1931).

[31] C. Catlett et al., in HPC and Grids in Action, edited by Luco Grandinetti (IOS Press, Amsterdam, 2007). 